\title{
THE LEADERSHIP STYLES OF SCHOOL OF PHYSICAL EDUCATION AND SPORTS IN DEPARTMENT OF COACHING EDUCATION STUDENTS ${ }^{1}$
}

\section{BEDEN EĞİTIMİ ve SPOR YÜKSEKOKULU ANTRENÖRLÜK EĞİTIMİ BÖLÜMÜ ÖĞRENCILLEIININ LIDERLIKK STILLERINIIN INNCELENMESI}

\author{
Bürke KÖKSALAN ${ }^{1}$, Nurper ÖZBAR ${ }^{1}$, Kürşat KARACABEY $Y^{1}$, Sevcan KARACABEY ${ }^{2}$ \\ ${ }^{1}$ Düzce University Vocational School of Physical Education and Sports, Düzce / Turkey \\ ${ }^{2}$ Gaziantep University Institute of Health Sciences Department of Histology Embryology, Gaziantep / Turkey
}

Öz: Amaç: Farklı yaş kategorilerindeki gruplara liderlik yapacak olan antrenörlük eğitimi bölümü ögrencilerinin iyi bir lider olabilmeleri, çalıştıları spor kulüplerinde üstlerine düșen görevleri en iyi şekilde yerine getirebilmeleri açısından önemlidir. Bu yüzden çalışmamız antrenörlük eğitimi bölümü ögrencilerinin liderlik tiplerini belirleyebilmek amacıyla yapılmıştır. Materyal ve Yöntem: Bu çalışma, tarama (survey) modeli niteliğindedir. Veriler, Marmara Üniversitesi Beden Eğitimi ve Spor Yüksekokulu'nda okuyan Antrenörlük Eğitimi Bölümü ögrencilerinin liderlik ile ilgili görüş ve düşünceleri alınarak toplanmıștır. Çalışmamıza, 60'ı male, 50'si kız toplam 110 öğrenci gönüllü katılmıştır. Veri toplama aracı olarak, Richard Daft (2008) tarafindan geliştirilen Liderlik Ölçeği kullanılmıştır. Antrenörlük Eğitimi Bölümü öğrencilerinin algılarına göre, davranışsal liderlik özelliklerinin farklılaşıp farklılaşmadığının incelenmesinde tanımlayıcı istatistik (frekans-yüzde) ve ki kare-testi kullanılmıștı. Araştırmada anlamllık düzeyi 0.05 olarak kabul edilmiştir. Bulgular: Antrenörlük eğitimi bölümü ögrencilerinin cinsiyetlerine göre liderlik davranışları incelendiğinde genel olarak insan odaklı ve görev odaklı liderlik tiplerinin ikisine de uydukları ve cinsiyetler arasında fark olmadı̆̆ı tespit edilmiștir ( $p>0.05)$. Branşlar arasındaki insan ve görev odaklı liderlik davranışııın puanlaması değerlendirildiğinde görev odaklı davranış tipinde branşlar arasında istatistiksel açıdan fark olmadığı $(\mathrm{p}>0.05)$, ancak insan odaklı davranış tipinde branşlar arasında istatistiksel açıdan anlamlı fark olduğu $(\mathrm{p}<0.05)$ belirlenmiştir. Sonuçlar: Antrenörlük eğitimi bölümü ögrencilerinin davranışsal liderlik stilinde görev odaklı ve insan odaklı liderlik özelliğini hemen hemen eşit oranlarda sergiledikleri ve cinsiyetler arasında fark olmadığı belirlenmiștir. Liderlik stillerinin branşlara göre farklılıklarının incelenmesi sonucunda ise bazı branşlarda fark olmasına rağmen genel ortalamayı bozmadığı ve her iki tip liderlik özelliğini de taşıdıkları tespit edilmiş̧tir. Bu tarz liderlik özelliği sportif anlamda başarının daha kolay ede edilmesini sağlayacağı düşünülmektedir.

Anahtar Kelimeler: Beden Eğitimi ve Spor, Antrenörlük Eğitimi, Davranışsal Liderlik, Liderlik Ölçeği
Abstract: Aim: It is really important for students of coaching education program who will lead different age groups in trainings to be good leaders in terms of accomplishing the tasks given by the sport clubs which they work in. Because of this, our research is done to find the leadership styles of students of coaching education program. Material and Methods: This research has the characteristic of survey model. Data is collected by asking the opinions and thoughts on leadership to the students from Marmara University School of Physical Education and Sports Department of Coaching Education. 110 students are participated in our research and while 60 of them are male students 50 of them are female. Leadership Questionnaire developed by Richard Daft (2008) is used as data collector. Descriptive statistics (frequency-percentage) and Chi-square-test are used to analyze if behavioral leadership qualifications become different or not according to perception of coaching education program. Results: When we analyze the leadership behaviors of coaching education students according to their gender, it is found that they are fit to both people oriented and job oriented leadership types and there is not a difference between genders $(p>0.05)$. When the scoring of people oriented and job oriented leadership behaviors between branches is reviewed, it is found that there is not a significant difference in job oriented behavior between branches ( $p>0.05$ ). However, a significant difference in people oriented behavior is found $(\mathrm{p}<0.05)$. Conclusion: It is found that Coaching Education students show equal rate of job oriented and people oriented leadership qualifications in behavioral leadership style and also it is found there is no difference between genders. In the result of analyzing the difference of leadership styles of according to branches, even though there are some differences between some branches, this difference does not disturb the general average and they carry two different type of leadership. This kind of leadership characteristics help to gain sportive success.

Key Words: Physical Education and Sport, Coaching Education, Behavioral Leadership, Leadership Scale

Doi: 10.17363/SSTB.20161816495

(1) Corresponding Author: Kürşat KARACABEY, Duzce University, School of Physical Education and Sports Düzce / Turkeykkaracabey@gmail.com Received: 19.12.2015 Accepted: 18.03.2016 Type ofarticle (Research-Application) Conflict of Interest: None / "None of Ethics Committee" 


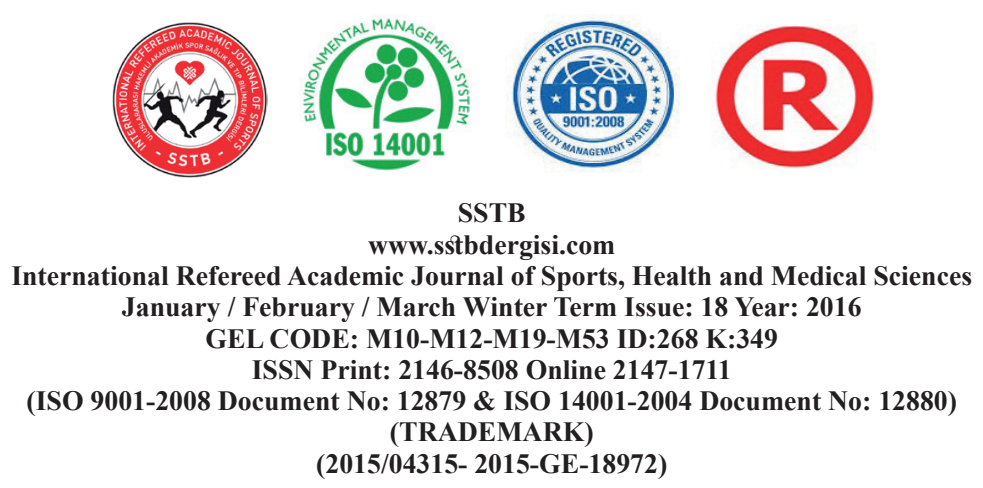

\section{INTRODUCTION}

Leaders have been subjects of many studies that have always attracted attention since they influence the lives of societies and change the course of history. Leadership enables the effective functioning of an organization by influencing people in order to achieve organizational goals (Aydın, 1994:1-2) Not all managers have leadership abilities, but being a good manager requires having a leadership ability (Sabuncuoğlu, 2001).

It has been discussed for a long time whether leadership can be taught or not. It has been determined that the leadership abilities can be taught. An increasing demand has emerged in leadership training and development programs (Mole, 2004). It has been determined that the current training programs for developing leadership skills are not adequate and need to be developed (Elmuti et al., 2005).

In explaining what qualities a successful leader should have, James and Eden (2001) mention the ability to see the future and to keep his/her horizons broad. They state that success-focused leaders proceed to achieve their targets, that they have the skill to always motivate others, that they should be guiding in how to reach a target they have set, and that following the achievement of the target, they should continue to back up the people they lead.
Behavioral approaches have been developed as a consequence of researchers' focusing on how leaders behave and what they do rather than concentrating on their personal characteristics because leadership analysis of the characteristics approach has quite shallow and reductionist aspects. The building block of this theory is constituted by the behaviors of the leaders rather than their personal characteristics. Therefore, the relationships of a leader with his/her audience are not based on leadership characteristics, but on whether his/her attitudes and behavior are accepted by his/her audience. Thus, leaders cannot be thought of separately and independently from the groups that they lead, and they must be evaluated in terms of their relationship with such groups (Gelatt, 2002).

This behavior-focused theory makes a distinction between effective and ineffective leaders. According to this theory, behaviors can be taught, and individuals can be trained and thus enabled to perform better leadership (Gelatt, 2002).

Successful leading coaches in sports recognize that the relationship between themselves and the environment positively affect the performance of athletes. Leadership in sports is an area that requires specialization in many personal and technical abilities. It is not an easy task to influence the actions and behaviors of different players in a team. Especially 


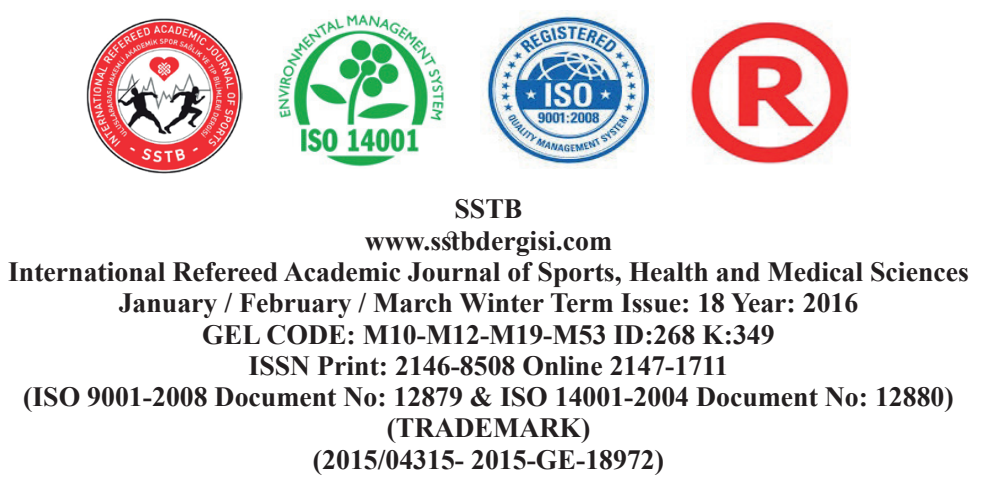

with the changes in the nature of sports in recent years, coaches have come to interact with many persons and groups (Donuk, 2007).

Those leaders who train teams should do their best in order to be perfect in every aspect. Perfect leadership and coaching is dependent on the ability to adequately use psychological skills and especially interpersonal communication skills (Konter, 1996).

Teachers of physical education and sports, coaches, and sports managers assume duties to achieve a certain goal by managing a group. The leadership behaviors of coaches, physical education teachers and sports managers resemble those leadership behaviors in other occupations (Laurent and Bradney, 2007).

Studies dealing with behavioral approach have determined that leadership behaviors are gathered around two main variables. These are the variable of initiating structure and the variable of consideration. The variable of initiating structure refers to task-related behaviors such as shaping the work environment, defining the responsibilities, and planning the tasks. The variable of consideration refers to the behaviors relate to individual relationships such as respect between leaders and followers, trust, and friendship. Based on these definitions, it can be suggested that the variable of initiating structure is related to the

needs of an enterprise while the variable of consideration is related to the needs of workers (Northouse, 2004).

In his study, Stodgill states that successful leaders should focus both on workers - i.e. they should increase workers' skills - and on tasks (Davis, 1997). Considering all the characteristics, the leadership styles of coaches may vary by the characteristics of the athletes in their team and by other external factors (Temel, 2010).

Prospective coaches are expected to exhibit leadership behavior in order to become successful in their occupation. Therefore, the acquisition of leadership behaviors by the students of School of Physical Education and Sports will enable them to be successful in their occupational lives. This research was conducted for the purpose of detecting the opinions of the students from the coaching department and to make recommendations based on the results of the research.

\section{MATERIAL and METHOD}

This research employed the survey method. The data were collected by getting the views and opinions of the students of the Department of Coaching Education of the School of Physical Education and Sports of Marmara University. 


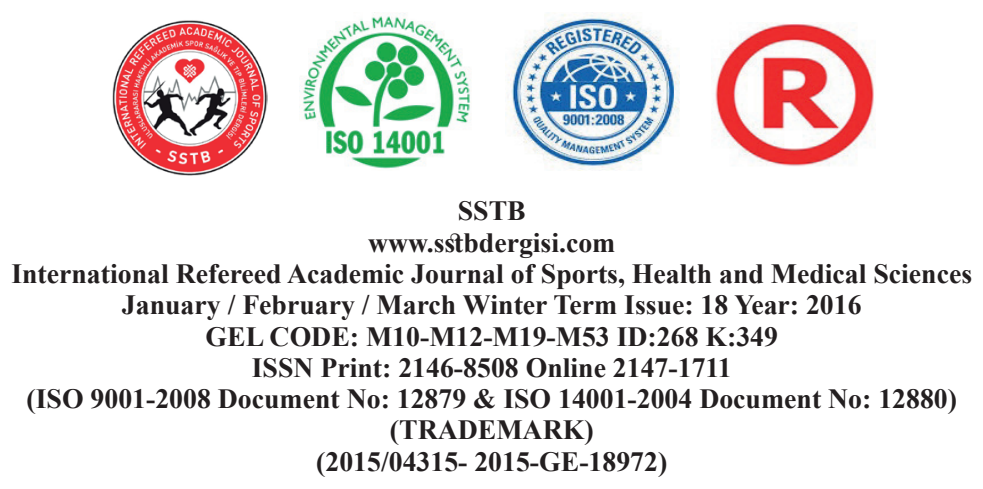

The research is significant for the students of the department of coaching education, who will be leading various groups with various age categories in the future, to become good leaders and to adequately fulfill their duties in the sports clubs they will work for.

\section{Respondents of the Questionnaire}

Students from the Department of Coaching Education of the School of Physical Education and Sports voluntarily participated in the research. Students were randomly selected.

\section{Research Population and Design}

The population of the research includes the students attending the School of Physical Education and Sports, and the sample is composed of 110 persons (60 males and 50 females) studying in the Department of Coaching Education. Questionnaire technique was employed for the research design.

\section{Data Collection}

Leadership Questionnaire, developed by Richard Daft (2008) was used as a data collection tool in the research. The questionnaire is made up of 38 questions; 3 of them are demographic questions, and 35 are Likerttype questions. Responses for the Likert-type questions were measured through a 5-point Likert scale $(1=$ strongly disagree; $5=$ strongly agree $)(\alpha=0.7627)$.
In the leadership scale developed by Richard Daft, the questions $1,2,4,6,8,9,11,12,13$, $14,16,17,20,21,23,25,27,29,30,31$ and 33 are related to task-oriented leadership, and the questions $3,5,7,10,15,18,19,22,24$, $26,28,32,34$ and 35 are related to peopleoriented leadership.

The questionnaire forms were filled in by the respondents themselves, who voluntarily took part in the study. An application was made to the Directorate of the School of Physical Education and Sports of Marmara University with the submission of the questionnaire form and the content of the study. The study was carried out with the permission of the directorate and with the support of the students from the mentioned department.

\section{Statistical Methods Used}

Descriptive statistics (frequency - percentage) and chi square test were used in the evaluation of the data obtained. The significance level was determined to be 0.05 in statistical calculations.

\section{FINDINGS}

The following tables present the findings related to the behavioral leadership skills of the students from the department of coaching education. 
www.sstbdergisi.com

International Refereed Academic Journal of Sports, Health and Medical Sciences January / February / March Winter Term Issue: 18 Year: 2016

GEL CODE: M10-M12-M19-M53 ID:268 K:349

ISSN Print: 2146-8508 Online 2147-1711

(ISO 9001-2008 Document No: 12879 \& ISO 14001-2004 Document No: 12880)

(TRADEMARK)

(2015/04315- 2015-GE-18972)

Table 1. \% Of Responses Related to Task-Oriented Leadership Behavior By Gender

\begin{tabular}{|c|c|c|c|c|c|c|c|c|c|c|c|c|}
\hline & \multirow{2}{*}{ QUESTION } & \multirow{2}{*}{ GENDER } & \multicolumn{2}{|c|}{ Never } & \multicolumn{2}{|c|}{ Seldom } & \multicolumn{2}{|c|}{ Occasionally } & \multicolumn{2}{|c|}{ Frequently } & \multicolumn{2}{|c|}{ Always } \\
\hline & & & $\mathrm{F}$ & $\%$ & $\mathrm{~F}$ & $\%$ & $\mathrm{~F}$ & $\%$ & $\mathrm{~F}$ & $\%$ & $\mathrm{~F}$ & $\%$ \\
\hline \multirow{2}{*}{1} & \multirow{2}{*}{$\begin{array}{l}\text { I would most likely act as the } \\
\text { spokesperson of the group }\end{array}$} & Female & 1 & 2,0 & 8 & 16,0 & 25 & 50,0 & 4 & 8,0 & 12 & 24,0 \\
\hline & & Male & 0 & 0 & 1 & 1,7 & 31 & 51,7 & 12 & 20,0 & 16 & 26,7 \\
\hline \multirow{2}{*}{2} & \multirow{2}{*}{$\begin{array}{l}\text { I would encourage overtime } \\
\text { work }\end{array}$} & Female & 16 & 32,0 & 7 & 14,0 & 17 & 34,0 & 7 & 14,0 & 3 & 6,0 \\
\hline & & Male & 10 & 16,7 & 9 & 15,0 & 20 & 33,3 & 7 & 11,7 & 14 & 23,3 \\
\hline \multirow{2}{*}{4} & \multirow{2}{*}{$\begin{array}{l}\text { I would encourage the use of } \\
\text { uniform procedures }\end{array}$} & Female & 2 & 4,0 & 1 & 2,0 & 17 & 34,0 & 8 & 16,0 & 22 & 44,0 \\
\hline & & Male & 2 & 3,3 & 7 & 11,7 & 19 & 31,7 & 16 & 26,7 & 16 & 26,7 \\
\hline \multirow{2}{*}{6} & \multirow{2}{*}{$\begin{array}{l}\text { I would stress being ahead of } \\
\text { competing groups }\end{array}$} & Female & 8 & 16,0 & 0 & 0 & 20 & 40,0 & 10 & 20,0 & 12 & 24,0 \\
\hline & & Male & 9 & 15,0 & 7 & 11,7 & 13 & 21,7 & 17 & 28,3 & 14 & 23,3 \\
\hline \multirow{2}{*}{8} & \multirow{2}{*}{$\begin{array}{l}\text { I would speak as a } \\
\text { representative of the group }\end{array}$} & Female & 0 & 0 & 9 & 18,0 & 15 & 30,0 & 12 & 24,0 & 14 & 28,0 \\
\hline & & Male & 0 & 0 & 3 & 5,0 & 21 & 35,0 & 21 & 35,0 & 15 & 25,0 \\
\hline \multirow{2}{*}{9} & \multirow{2}{*}{$\begin{array}{l}\text { I would try out my ideas in the } \\
\text { group }\end{array}$} & Female & 3 & 6,0 & 8 & 16,0 & 1 & 2,0 & 24 & 48,0 & 14 & 28,0 \\
\hline & & Male & 0 & 0 & 10 & 16,7 & 14 & 23,3 & 20 & 33,3 & 16 & 26,7 \\
\hline \multirow{2}{*}{11} & \multirow{2}{*}{$\begin{array}{l}\text { I would be working hard for a } \\
\text { promotion }\end{array}$} & Female & 1 & 2,0 & 1 & 2,0 & 8 & 16,0 & 18 & 36,0 & 22 & 44,0 \\
\hline & & Male & 0 & 0 & 7 & 11,7 & 5 & 8,3 & 18 & 30,0 & 30 & 50,0 \\
\hline \multirow{2}{*}{12} & \multirow{2}{*}{$\begin{array}{l}\text { I would tolerate postponement } \\
\text { and uncertainty }\end{array}$} & Female & 21 & 42,0 & 11 & 22,0 & 10 & 20,0 & 8 & 16,0 & 0 & 0 \\
\hline & & Male & 29 & 48,3 & 13 & 21,7 & 12 & 20,0 & 5 & 8,3 & 1 & 1,7 \\
\hline \multirow{2}{*}{13} & \multirow{2}{*}{$\begin{array}{l}\text { I would speak for the group if } \\
\text { visitors were present }\end{array}$} & Female & 2 & 4,0 & 10 & 20,0 & 11 & 22,0 & 17 & 34,0 & 10 & 20,0 \\
\hline & & Male & 7 & 11,7 & 4 & 6,7 & 25 & 41,7 & 13 & 21,7 & 11 & 18,3 \\
\hline \multirow{2}{*}{14} & I would keen the work moving & Female & 0 & 0 & 0 & 0 & 8 & 16,0 & 10 & 20,0 & 32 & 64,0 \\
\hline & at a rapid pace & Male & 2 & 3,3 & 1 & 1,7 & 7 & 11,7 & 18 & 30,0 & 32 & 53,3 \\
\hline 16 & I would settle conflicts when & Female & 0 & 0 & 0 & 0 & 5 & 10,0 & 31 & 62,0 & 14 & 28,0 \\
\hline 10 & they occur in the group & Male & 0 & 0 & 2 & 3,3 & 10 & 16,7 & 19 & 31,7 & 29 & 48,3 \\
\hline 17 & I would ot curommed by & Female & 3 & 6,0 & 3 & 6,0 & 27 & 54,0 & 13 & 26,0 & 4 & 8,0 \\
\hline 17 & I would get swamped b & Male & 6 & 10,0 & 14 & 23,3 & 21 & 35,0 & 16 & 26,7 & 3 & 5,0 \\
\hline 20 & I would decide what should be done & ne Female & 2 & 4,0 & 9 & 18,0 & 10 & 20,0 & 17 & 34,0 & 12 & 24,0 \\
\hline 20 & and how it should be done & Male & 0 & 0 & 3 & 5,0 & 13 & 21,7 & 26 & 43,3 & 18 & 30,0 \\
\hline 21 & I would push for increased & Female & 0 & 0 & 5 & 10,0 & 16 & 32,0 & 17 & 34,0 & 12 & 24,0 \\
\hline 21 & production & Male & 0 & 0 & 0 & 0 & 13 & 21,7 & 28 & 46,7 & 19 & 31,7 \\
\hline 23 & Things would usually turn out as I & Female & 0 & 0 & 1 & 2,0 & 15 & 30,0 & 21 & 42,0 & 13 & 26,0 \\
\hline 23 & had predicted & Male & 0 & 0 & 0 & 0 & 7 & 11,7 & 30 & 50,0 & 23 & 38,3 \\
\hline 25 & I would assign group members to & Female & 5 & 10,0 & 0 & 0 & 20 & 40,0 & 14 & 28,0 & 11 & 22,0 \\
\hline 25 & particular tasks & Male & 11 & 18,3 & 6 & 10,0 & 21 & 35,0 & 14 & 23,3 & 8 & 13,3 \\
\hline
\end{tabular}




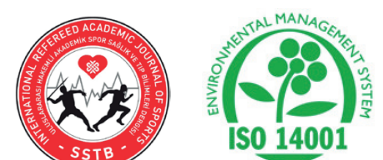

SSTB

www.sstbdergisi.com

International Refereed Academic Journal of Sports, Health and Medical Sciences January / February / March Winter Term Issue: 18 Year: 2016

GEL CODE: M10-M12-M19-M53 ID:268 K:349

ISSN Print: 2146-8508 Online 2147-1711

(ISO 9001-2008 Document No: 12879 \& ISO 14001-2004 Document No: 12880)

(TRADEMARK)

(2015/04315- 2015-GE-18972)

\begin{tabular}{lllrrrrrrrrrr}
27 & I would ask the members to work \\
harder & Female & 8 & 16,0 & 13 & 26,0 & 21 & 42,0 & 7 & 14,0 & 1 & 2,0 \\
& Male & 4 & 6,7 & 12 & 20,0 & 13 & 21,7 & 18 & 30,0 & 13 & 21,7 \\
$\begin{array}{l}\text { I would schedule the work to be } \\
\text { done }\end{array}$ & Female & 0 & 0 & 3 & 6,0 & 16 & 32,0 & 15 & 30,0 & 16 & 32,0 \\
& Male & 0 & 0 & 2 & 3,3 & 10 & 16,7 & 27 & 45,0 & 21 & 35,0 \\
$\mathbf{3 0}$ & $\begin{array}{l}\text { I would refuse to explain my } \\
\text { actions }\end{array}$ & Female & 9 & 18,0 & 8 & 16,0 & 16 & 32,0 & 12 & 24,0 & 5 & 10,0 \\
$\mathbf{3 1}$ & Male & 9 & 15,0 & 15 & 25,0 & 20 & 33,3 & 15 & 25,0 & 1 & 1,7 \\
$\begin{array}{l}\text { I would persuade others that my } \\
\text { ideas are to their advantage }\end{array}$ & Female & 1 & 2,0 & 6 & 12,0 & 19 & 38,0 & 17 & 34,0 & 7 & 14,0 \\
& Male & 0 & 0 & 7 & 11,7 & 15 & 25,0 & 27 & 45,0 & 11 & 18,3 \\
$\mathbf{3 3}$ & $\begin{array}{l}\text { I would urge the group to beat its } \\
\text { previous record }\end{array}$ & Female & 7 & 14,0 & 1 & 2,0 & 13 & 26,0 & 21 & 42,0 & 8 & 16,0 \\
& Male & 2 & 3,3 & 2 & 3,3 & 10 & 16,7 & 26 & 43,3 & 20 & 33,3 \\
\hline
\end{tabular}

$\mathrm{X} 2=5.071, \mathrm{P}=.496, \mathrm{P}>.05$

No significant difference by gender was

found with respect to the task-oriented lead-

ership behavior. 

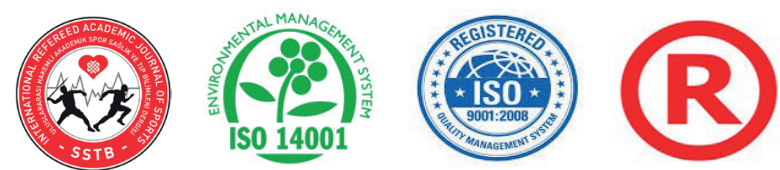

SSTB

www.sstbdergisi.com

International Refereed Academic Journal of Sports, Health and Medical Sciences January / February / March Winter Term Issue: 18 Year: 2016

GEL CODE: M10-M12-M19-M53 ID:268 K:349

ISSN Print: 2146-8508 Online 2147-1711

(ISO 9001-2008 Document No: 12879 \& ISO 14001-2004 Document No: 12880)

(TRADEMARK)

(2015/04315- 2015-GE-18972)

Table 2. \% Of Responses Related to People-Oriented Leadership Behavior By Gender

\begin{tabular}{|c|c|c|c|c|c|c|c|c|c|c|c|c|}
\hline \multirow{2}{*}{\multicolumn{2}{|c|}{ QUESTION }} & \multirow{2}{*}{ GENDER } & \multicolumn{2}{|c|}{ Never } & \multicolumn{2}{|c|}{ Seldom } & \multicolumn{2}{|c|}{ Occasionally } & \multicolumn{2}{|c|}{ Frequently } & \multicolumn{2}{|c|}{ Always } \\
\hline & & & $\mathrm{F}$ & $\%$ & $\mathrm{~F}$ & $\%$ & $\mathrm{~F}$ & $\%$ & $\mathrm{~F}$ & $\%$ & $\mathrm{~F}$ & $\%$ \\
\hline \multirow[t]{2}{*}{3} & \multirow{2}{*}{$\begin{array}{l}\text { I would allow members complete } \\
\text { freedom in their work }\end{array}$} & Female & 7 & 14,0 & 18 & 36,0 & 10 & 20,0 & 7 & 14,0 & 8 & 16 , \\
\hline & & Male & 9 & 15,0 & 12 & 20,0 & 23 & 38,3 & 11 & 18,3 & 5 & 8 \\
\hline \multirow[t]{2}{*}{5} & \multirow{2}{*}{$\begin{array}{l}\text { I would permit members to use their } \\
\text { own judgment in solving problems }\end{array}$} & Femal & 3 & 6,0 & 5 & 10,0 & 15 & 30,0 & 11 & 22,0 & 16 & 32, \\
\hline & & 90 & 1 & 1,7 & 4 & 6,7 & 25 & 41,7 & 18 & 30,0 & 12 & 20 \\
\hline \multirow[t]{2}{*}{7} & \multirow{2}{*}{$\begin{array}{l}\text { I would needle members for greater } \\
\text { effort }\end{array}$} & Fem & 8 & 16,0 & 16 & 32,0 & 4 & 8,0 & 16 & 32,0 & 6 & 12 \\
\hline & & M & 4 & 6,7 & 4 & 6,7 & 25 & 41,7 & 0 & 0 & 0 & \\
\hline \multirow[t]{2}{*}{10} & \multirow{2}{*}{$\begin{array}{l}\text { I would let members do their work the } \\
\text { way they think best }\end{array}$} & Fem & 0 & 0 & 2 & 4,0 & 16 & 32,0 & 16 & 32,0 & 16 & 32 \\
\hline & & Mal & J & 5,0 & 0 & 0 & 21 & 0 & 27 & 45,0 & 9 & \\
\hline \multirow[t]{2}{*}{15} & \multirow{2}{*}{$\begin{array}{l}\text { I would turn the members loose on a } \\
\text { job and let them go to it }\end{array}$} & & 0 & 0 & 1 & 2,0 & 1 & 2,0 & 15 & 30,0 & 33 & 66 , \\
\hline & & & 1 & 1,7 & 4 & 6,7 & 1 & 1,7 & 31 & 51,7 & 23 & 38 \\
\hline \multirow[t]{2}{*}{18} & \multirow{2}{*}{$\begin{array}{l}\text { I would represent the group at outside } \\
\text { meetings }\end{array}$} & & 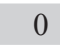 & 0 & 9 & 18,0 & 28 & 56,0 & 7 & 14,0 & 6 & 12 \\
\hline & & & 2 & 3,3 & 7 & & 20 & 3 & 22 & 36,7 & 9 & 15 \\
\hline \multirow[t]{2}{*}{19} & \multirow{2}{*}{$\begin{array}{l}\text { I would be reluctant to allow the } \\
\text { members any freedom of action }\end{array}$} & & 11 & 22,0 & 22 & 44,0 & 7 & 14,0 & 9 & 18,0 & 1 & 2 , \\
\hline & & & 22 & 36,7 & 11 & 18,3 & 20 & & 7 & 11,7 & 0 & \\
\hline \multirow[t]{2}{*}{22} & \multirow{2}{*}{$\begin{array}{l}\text { I would let some members have } \\
\text { authority that I could keep }\end{array}$} & & 2 & 4,0 & 1 & 2,0 & 26 & 52,0 & 16 & 32,0 & 5 & 10 \\
\hline & & & 2 & 3,3 & 6 & 10,0 & 22 & 36,7 & 14 & 23,3 & 16 & 26,7 \\
\hline \multirow[t]{2}{*}{24} & \multirow{2}{*}{$\begin{array}{l}\text { I would allow the group a high degree } \\
\text { of initiative }\end{array}$} & Fen & 2 & 4,0 & 1 & 2,0 & 17 & 34,0 & 24 & 48,0 & 6 & 12 \\
\hline & & & ( & 5,0 & 3 & 5,0 & 24 & 40,0 & 21 & 35,0 & 9 & 15,0 \\
\hline \multirow[t]{2}{*}{26} & \multirow{2}{*}{ I would be willing to make changes } & Fen & 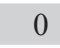 & 0 & 4 & 8,0 & 16 & 32,0 & 10 & 20,0 & 20 & 40 , \\
\hline & & $\mathrm{Ma}$ & 0 & 0 & 9 & 15,0 & 17 & 28,3 & 11 & 18,3 & 23 & 38,3 \\
\hline \multirow[t]{2}{*}{28} & & Fer & 0 & 4,0 & 2 & 4,0 & 13 & 26,0 & 16 & 32,0 & 17 & 34, \\
\hline & & Mal & 0 & 0 & 1 & 1,7 & 33 & 55,0 & 18 & 30,0 & 8 & 13,3 \\
\hline 32 & uld nermit the oroun to set its & Femal & 0 & 0 & 3 & 6,0 & 6 & 12,0 & 24 & 48,0 & 17 & 34, \\
\hline & & Male & 5 & 8,3 & 1 & 1,7 & 17 & 28,3 & 23 & 38,3 & 14 & 23,3 \\
\hline 34 & I would act without consulting the & Femal & 33 & 66,0 & 3 & 6,0 & 6 & 12,0 & 5 & 10,0 & 3 & 6 , \\
\hline & & Male & 11 & 18,3 & 19 & 31,7 & 17 & 28,3 & 5 & 8,3 & 8 & 13,3 \\
\hline 35 & I would ask tl & & 0 & 0 & 3 & 6,0 & 3 & 6,0 & 19 & 38,0 & 25 & 50 \\
\hline & & Male & 4 & 6,7 & 2 & 3,3 & 8 & 13,3 & 23 & 38,3 & 23 & 38,3 \\
\hline
\end{tabular}

$\mathrm{X} 2=4.103, \mathrm{P}=.105, \mathrm{P}>.05$

No significant difference by gender was

found with respect to the people-oriented

leadership behavior. 
www.sstbdergisi.com

International Refereed Academic Journal of Sports, Health and Medical Sciences January / February / March Winter Term Issue: 18 Year: 2016

GEL CODE: M10-M12-M19-M53 ID:268 K:349

ISSN Print: 2146-8508 Online 2147-1711

(ISO 9001-2008 Document No: 12879 \& ISO 14001-2004 Document No: 12880)

(TRADEMARK)

(2015/04315- 2015-GE-18972)

Table 3. \% Distribution of Responses Related to Task-Oriented Leadership Behavior By Branch

\begin{tabular}{|c|c|c|c|c|c|c|c|c|c|c|c|c|}
\hline \multirow{2}{*}{\multicolumn{2}{|c|}{ QUESTION }} & \multirow{2}{*}{ BRANCH } & \multicolumn{2}{|c|}{ Never } & \multicolumn{2}{|c|}{ Seldom } & \multicolumn{2}{|c|}{ Occasionally } & \multicolumn{2}{|c|}{ Frequently } & \multicolumn{2}{|c|}{ Always } \\
\hline & & & $\mathrm{F}$ & $\%$ & $\mathrm{~F}$ & $\%$ & $\mathrm{~F}$ & $\%$ & $\mathrm{~F}$ & $\%$ & $\mathrm{~F}$ & $\%$ \\
\hline \multirow{5}{*}{1} & \multirow{5}{*}{$\begin{array}{l}\text { I would most likely act as the } \\
\text { spokesperson of the group }\end{array}$} & Football & $\overline{0}$ & 0 & 0 & 0 & 11 & 50.0 & 7 & 31.8 & 4 & 18.2 \\
\hline & & Handball & 0 & 0 & 0 & 0 & 16 & 72.7 & 1 & 4.5 & 5 & 22.7 \\
\hline & & Basketball & 0 & 0 & 6 & 27.3 & 7 & 31.8 & 4 & 18.2 & 5 & 22.7 \\
\hline & & Volleyball & 0 & 0 & 2 & 9.1 & 13 & 59.1 & 0 & 0 & 7 & 31.8 \\
\hline & & Individual & 1 & 4.5 & 1 & 4.5 & 9 & 40.9 & 4 & 18.2 & 7 & 31.8 \\
\hline \multirow{5}{*}{2} & \multirow{5}{*}{ I would encourage overtime work } & Football & 3 & 13.6 & 1 & 4.5 & 8 & 36.4 & 5 & 22.7 & 5 & 22.7 \\
\hline & & Handball & 8 & 36.4 & 4 & 18.2 & 6 & 27.3 & 0 & 0 & 4 & 18.2 \\
\hline & & Basketball & 7 & 31.8 & 0 & 0 & 13 & 59.1 & 2 & 9.1 & 0 & 0 \\
\hline & & Volleyball & 3 & 13.6 & 6 & 27.3 & 2 & 9.1 & 4 & 18.2 & 7 & 31.8 \\
\hline & & Individual & 5 & 22.7 & 5 & 22.7 & 8 & 36.4 & 3 & 13.6 & 1 & 4.5 \\
\hline \multirow{5}{*}{4} & \multirow{5}{*}{$\begin{array}{l}\text { I would encourage the use of } \\
\text { uniform procedures }\end{array}$} & Football & 0 & 0 & 1 & 4.5 & 8 & 36.4 & 7 & 31.8 & 6 & 27.3 \\
\hline & & Handball & 0 & 0 & 3 & 13.6 & 7 & 31.8 & 3 & 13.6 & 9 & 40.9 \\
\hline & & Basketball & 2 & 9.1 & 2 & 9.1 & 5 & 22.7 & 0 & 0 & 13 & 59.1 \\
\hline & & Volleyball & 2 & 9.1 & 0 & 0 & 13 & 59.1 & 4 & 18.2 & 3 & 13.6 \\
\hline & & Individual & 0 & 0 & 2 & 9,1 & 3 & 13.6 & 10 & 45.5 & 7 & 31.8 \\
\hline \multirow{5}{*}{6} & \multirow{5}{*}{$\begin{array}{l}\text { I would stress being ahead of } \\
\text { competing groups }\end{array}$} & Football & 5 & 22.7 & 3 & 13.6 & 2 & 9.1 & 6 & 27.3 & 6 & 27.3 \\
\hline & & Handball & 0 & 0 & 0 & 0 & 2 & 9.1 & 8 & 36.4 & 12 & 54.5 \\
\hline & & Basketball & 2 & 9.1 & 0 & 0 & 12 & 54.5 & 8 & 36.4 & 0 & 0 \\
\hline & & Volleyball & 4 & 18.2 & 0 & 0 & 9 & 40.9 & 2 & 9.1 & 7 & 31.8 \\
\hline & & Individual & 6 & 27.3 & 4 & 18.2 & 8 & 36.4 & 3 & 13.6 & 1 & 4.5 \\
\hline \multirow{5}{*}{8} & \multirow{5}{*}{$\begin{array}{l}\text { I would speak as a representative } \\
\text { of the group }\end{array}$} & Football & 0 & 0 & 2 & 9.1 & 9 & 40.9 & 5 & 22.7 & 6 & 27.3 \\
\hline & & Handball & 0 & 0 & 0 & 0 & 6 & 27.3 & 12 & 54.5 & 4 & 18.2 \\
\hline & & Basketball & 0 & 0 & 6 & 27.3 & 6 & 27.3 & 3 & 13.6 & 7 & 31.8 \\
\hline & & Volleyball & 0 & 0 & 0 & 0 & 6 & 27.3 & 9 & 40.9 & 7 & 31.8 \\
\hline & & Individual & 0 & 0 & 4 & 18.2 & 9 & 40.9 & 4 & 18.2 & 5 & 22.7 \\
\hline \multirow{5}{*}{9} & \multirow{5}{*}{$\begin{array}{l}\text { I would try out my ideas in the } \\
\text { group }\end{array}$} & Football & 0 & 0 & 2 & 9,1 & 5 & 22,7 & 11 & 50,0 & 4 & 18,2 \\
\hline & & Handball & 1 & 4,5 & 6 & 27,3 & 2 & 9,1 & 6 & 27,3 & 7 & 31,8 \\
\hline & & Basketball & 0 & 0 & 8 & 36,4 & 2 & 9,1 & 7 & 31,8 & 5 & 22,7 \\
\hline & & Volleyball & 0 & 0 & 0 & 0 & 0 & 0 & 15 & 68,2 & 7 & 31,8 \\
\hline & & Individual & 2 & 9,1 & 2 & 9,1 & 6 & 27,3 & 5 & 22,7 & 7 & 31,8 \\
\hline \multirow{5}{*}{11} & \multirow{5}{*}{$\begin{array}{l}\text { I would be working hard for a } \\
\text { promotion }\end{array}$} & Football & 0 & 0 & 1 & 4,5 & 1 & 4,5 & 7 & 31,8 & 13 & 59,1 \\
\hline & & Handball & 0 & 0 & 4 & 18,2 & 3 & 13,6 & 11 & 50,0 & 4 & 18,2 \\
\hline & & Basketball & 0 & 0 & 2 & 9,1 & 0 & 0 & 13 & 59,1 & 7 & 31,8 \\
\hline & & Volleyball & 0 & 0 & 0 & 0 & 6 & 27,3 & 3 & 13,6 & 13 & 59,1 \\
\hline & & Individual & 1 & 4,5 & 1 & 4,5 & 3 & 13,6 & 2 & 9,1 & 15 & 68,2 \\
\hline
\end{tabular}


SSTB

www.sstbdergisi.com

International Refereed Academic Journal of Sports, Health and Medical Sciences January / February / March Winter Term Issue: 18 Year: 2016

GEL CODE: M10-M12-M19-M53 ID:268 K:349

ISSN Print: 2146-8508 Online 2147-1711

(ISO 9001-2008 Document No: 12879 \& ISO 14001-2004 Document No: 12880)

(TRADEMARK)

(2015/04315- 2015-GE-18972)

12

I would tolerate postponement and uncertainty

13

I would speak for the group if visitors were present

16

I would settle conflicts when they occur in the group

17

I would get swamped by details

I would decide what should

20

done

I would push for increased production

I would keep the work moving

$\begin{array}{lllllllllll}\text { Football } & 14 & 63,6 & 4 & 18,2 & 2 & 9,1 & 2 & 9,1 & 0 & 0\end{array}$

$\begin{array}{lllllllllll}\text { Handball } & 6 & 27,3 & 7 & 31,8 & 6 & 27,3 & 3 & 13,6 & 0 & 0\end{array}$

$\begin{array}{lllllllllll}\text { Basketball } & 15 & 68,2 & 2 & 9,1 & 5 & 22,7 & 0 & 0 & 0 & 0\end{array}$

$\begin{array}{lllllllllll}\text { Volleyball } & 7 & 31,8 & 7 & 31,8 & 0 & 0 & 8 & 36,4 & 0 & 0\end{array}$

$\begin{array}{lllllllllll}\text { Individual } & 8 & 36,4 & 4 & 18,2 & 9 & 40,9 & 0 & 0 & 1 & 4,5\end{array}$

$\begin{array}{lllllllllll}\text { Football } & 0 & 0 & 1 & 4,5 & 8 & 36,4 & 8 & 36,4 & 5 & 22,7\end{array}$

$\begin{array}{lllllllllll}\text { Handball } & 1 & 4,5 & 5 & 22,7 & 7 & 31,8 & 5 & 22,7 & 4 & 18,2\end{array}$

$\begin{array}{lllllllllll}\text { Basketball } & 3 & 13,6 & 6 & 27,3 & 4 & 18,2 & 4 & 18,2 & 5 & 22,7\end{array}$

$\begin{array}{lllllllllll}\text { Volleyball } & 4 & 18,2 & 2 & 9,1 & 4 & 18,2 & 9 & 40,9 & 3 & 13,6\end{array}$

$\begin{array}{lllllllllll}\text { Individual } & 1 & 4,5 & 0 & 0 & 13 & 59,1 & 4 & 18,2 & 4 & 18,2\end{array}$

$\begin{array}{lllllllllll}\text { Football } & 0 & 0 & 1 & 4,5 & 1 & 4,5 & 6 & 27,3 & 14 & 63,6\end{array}$

$\begin{array}{lrrrrrrrrrr}\text { Handball } & 0 & 0 & 0 & 0 & 4 & 18,2 & 3 & 13,6 & 15 & 68,2 \\ \text { Basketball } & 2 & 9,1 & 0 & 0 & 3 & 13,6 & 2 & 9,1 & 15 & 68,2\end{array}$

$\begin{array}{lllllllllll}\text { Volleyball } & 0 & 0 & 0 & 0 & 6 & 27,3 & 9 & 40,9 & 7 & 31,8\end{array}$

$\begin{array}{lllllllllll}\text { Individual } & 0 & 0 & 0 & 0 & 1 & 4,5 & 8 & 36,4 & 13 & 59,1\end{array}$

$\begin{array}{lllllllllll}\text { Football } & 0 & 0 & 0 & 0 & 2 & 9,1 & 9 & 40,9 & 11 & 50,0\end{array}$

$\begin{array}{lllllllllll}\text { Handball } & 0 & 0 & 2 & 9,1 & 6 & 27,3 & 7 & 31,8 & 7 & 31,8\end{array}$

$\begin{array}{lllllllllll}\text { Basketball } & 0 & 0 & 0 & 0 & 0 & 0 & 13 & 59,1 & 9 & 40,9\end{array}$

$\begin{array}{lllllllllll}\text { Volleyball } & 0 & 0 & 0 & 0 & 0 & 0 & 17 & 77,3 & 5 & 22,7\end{array}$

$\begin{array}{lrrrrrrrrrr}\text { Individual } & 0 & 0 & 0 & 0 & 7 & 31,8 & 4 & 18,2 & 11 & 50,0\end{array}$

$\begin{array}{lllllllllll}\text { Football } & 1 & 4,5 & 8 & 36,4 & 6 & 27,3 & 4 & 18,2 & 3 & 13,6\end{array}$

$\begin{array}{lllllllllll}\text { Handball } & 1 & 4,5 & 4 & 18,2 & 9 & 40,9 & 5 & 22,7 & 3 & 13,6\end{array}$

$\begin{array}{lllllllllll}\text { Basketball } & 0 & 0 & 2 & 9,1 & 10 & 45,5 & 10 & 45,5 & 0 & 0\end{array}$

$\begin{array}{lllllllllll}\text { Volleyball } & 4 & 18,2 & 0 & 0 & 12 & 54,5 & 6 & 27,3 & 0 & 0\end{array}$

$\begin{array}{lllllllllll}\text { Individual } & 3 & 13,6 & 3 & 13,6 & 11 & 50,0 & 4 & 18,2 & 1 & 4,5\end{array}$

$\begin{array}{lllllllllll}\text { Football } & 2 & 9,1 & 1 & 4,5 & 5 & 22,7 & 7 & 31,8 & 7 & 31,8\end{array}$

$\begin{array}{lllllllllll}\text { Handball } & 0 & 0 & 1 & 4,5 & 10 & 45,5 & 8 & 36,4 & 3 & 13,6\end{array}$

$\begin{array}{lllllllllll}\text { Basketball } & 0 & 0 & 8 & 36,4 & 0 & 0 & 5 & 22,7 & 9 & 40,9\end{array}$

\begin{tabular}{|c|c|c|c|c|c|}
\hline & \multicolumn{2}{|c|}{0} & 0 & 0 & 0 \\
\hline
\end{tabular}

$\begin{array}{lllllllllll}\text { Individual } & 0 & 0 & 2 & 9,1 & 8 & 36,4 & 8 & 36,4 & 4 & 18,2\end{array}$

$\begin{array}{lllllllllll}\text { Football } & 0 & 0 & 0 & 0 & 4 & 18,2 & 9 & 40,9 & 9 & 40,9\end{array}$

$\begin{array}{lrrrrrrrrrr}\text { Handball } & 0 & 0 & 1 & 4,5 & 8 & 36,4 & 11 & 50,0 & 2 & 9,1 \\ \text { Basketball } & 0 & 0 & 0 & 0 & 8 & 36,4 & 7 & 31,8 & 7 & 31,8\end{array}$

$\begin{array}{lllllllllll}\text { Volleyball } & 0 & 0 & 2 & 9,1 & 0 & 0 & 13 & 59,1 & 7 & 31,8\end{array}$

$\begin{array}{lllllllllll}\text { Individual } & 0 & 0 & 2 & 9,1 & 9 & 40,9 & 5 & 22,7 & 6 & 27,3\end{array}$



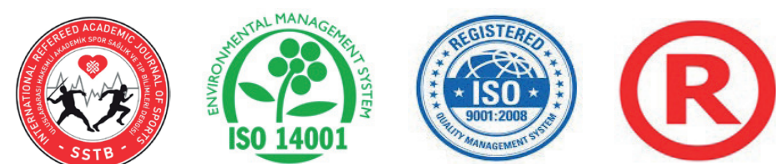

SSTB

www.sstbdergisi.com

International Refereed Academic Journal of Sports, Health and Medical Sciences January / February / March Winter Term Issue: 18 Year: 2016

GEL CODE: M10-M12-M19-M53 ID:268 K:349

ISSN Print: 2146-8508 Online 2147-1711

(ISO 9001-2008 Document No: 12879 \& ISO 14001-2004 Document No: 12880)

(TRADEMARK)

(2015/04315- 2015-GE-18972)

\begin{tabular}{|c|c|c|c|c|c|c|c|c|c|c|c|c|}
\hline \multirow{5}{*}{23} & \multirow{5}{*}{$\begin{array}{l}\text { Things would usually turn out } \\
\text { as I had predicted }\end{array}$} & Football & 0 & 0 & 0 & 0 & 2 & 9,1 & 10 & 45,5 & 10 & 45,5 \\
\hline & & Handball & 0 & 0 & 1 & 4,5 & 5 & 22,7 & 11 & 50,0 & 5 & 22,7 \\
\hline & & Basketball & 0 & 0 & 0 & 0 & 6 & 27,3 & 9 & 40,9 & 7 & 31,8 \\
\hline & & Volleyball & 0 & 0 & 0 & 0 & 0 & 0 & 13 & 59,1 & 9 & 40,9 \\
\hline & & Individual & 0 & 0 & 0 & 0 & 9 & 40,9 & 8 & 36,4 & 5 & 22,7 \\
\hline \multirow{5}{*}{25} & \multirow{5}{*}{$\begin{array}{l}\text { I would assign group members } \\
\text { to particular tasks }\end{array}$} & Football & 3 & 13,6 & 0 & 0 & 4 & 18,2 & 10 & 45,5 & 5 & 22,7 \\
\hline & & Handball & 2 & 9,1 & 3 & 13,6 & 12 & 54,5 & 1 & 4,5 & 4 & 18,2 \\
\hline & & Basketball & 4 & 18,2 & 2 & 9,1 & 5 & 22,7 & 11 & 50,0 & 0 & 0 \\
\hline & & Volleyball & 4 & 18,2 & 0 & 0 & 8 & 36,4 & 4 & 18,2 & 6 & 27,3 \\
\hline & & Individual & 3 & 13,6 & 1 & 4,5 & 12 & 54,5 & 2 & 9,1 & 4 & 18,2 \\
\hline \multirow{5}{*}{27} & \multirow{5}{*}{$\begin{array}{l}\text { I would ask the members to } \\
\text { work harder }\end{array}$} & Football & 4 & 18,2 & 7 & 31,8 & 6 & 27,3 & 4 & 18,2 & 1 & 4,5 \\
\hline & & Handball & 2 & 9,1 & 7 & 31,8 & 1 & 4,5 & 8 & 36,4 & 4 & 18,2 \\
\hline & & Basketball & 2 & 9,1 & 0 & 0 & 15 & 68,2 & 2 & 9,1 & 3 & 13,6 \\
\hline & & Volleyball & 0 & 0 & 3 & 13,6 & 4 & 18,2 & 11 & 50,0 & 4 & 18,2 \\
\hline & & Individual & 4 & 18,2 & 8 & 36,4 & 8 & 36,4 & 0 & 0 & 2 & 9,1 \\
\hline \multirow{5}{*}{29} & \multirow{5}{*}{$\begin{array}{l}\text { I would schedule the work to } \\
\text { be done }\end{array}$} & Football & 0 & 0 & 1 & 4,5 & 6 & 27,3 & 8 & 36,4 & 7 & 31,8 \\
\hline & & Handball & 0 & 0 & 1 & 4,5 & 8 & 36,4 & 12 & 54,5 & 1 & 4,5 \\
\hline & & Basketball & 0 & 0 & 0 & 0 & 6 & 27,3 & 7 & 31,8 & 9 & 40,9 \\
\hline & & Volleyball & 0 & 0 & 0 & 0 & 2 & 9,1 & 6 & 27,3 & 14 & 63,6 \\
\hline & & Individual & 0 & 0 & 3 & 13,6 & 4 & 18,2 & 9 & 40,9 & 6 & 27,3 \\
\hline \multirow{5}{*}{30} & \multirow{5}{*}{$\begin{array}{l}\text { I would refuse to explain my } \\
\text { actions }\end{array}$} & Football & 6 & 27,3 & 8 & 36,4 & 3 & 13,6 & 2 & 9,1 & 3 & 13,6 \\
\hline & & Handball & 4 & 18,2 & 1 & 4,5 & 13 & 59,1 & 4 & 18,2 & 0 & 0 \\
\hline & & Basketball & 0 & 0 & 2 & 9,1 & 4 & 18,2 & 16 & 72,7 & 0 & 0 \\
\hline & & Volleyball & 3 & 13,6 & 4 & 18,2 & 10 & 45,5 & 3 & 13,6 & 2 & 9,1 \\
\hline & & Individual & 5 & 22,7 & 8 & 36,4 & 6 & 27,3 & 2 & 9,1 & 1 & 4,5 \\
\hline \multirow{5}{*}{31} & \multirow{5}{*}{$\begin{array}{l}\text { I would persuade others that } \\
\text { my ideas are to their advantage }\end{array}$} & Football & 0 & 0 & 1 & 4,5 & 7 & 31,8 & 9 & 40,9 & 5 & 22,7 \\
\hline & & Handball & 0 & 0 & 3 & 13,6 & 5 & 22,7 & 11 & 50,0 & 3 & 13,6 \\
\hline & & Basketball & 0 & 0 & 8 & 36,4 & 5 & 22,7 & 6 & 27,3 & 3 & 13,6 \\
\hline & & Volleyball & 0 & 0 & 0 & 0 & 6 & 27,3 & 13 & 59,1 & 3 & 13,6 \\
\hline & & Individual & 1 & 4,5 & 1 & 4,5 & 11 & 50,0 & 5 & 22,7 & 4 & 18,2 \\
\hline \multirow[t]{5}{*}{33} & \multirow{5}{*}{$\begin{array}{l}\text { I would urge the group to beat } \\
\text { its previous record }\end{array}$} & Football & 4 & 18,2 & 1 & 4,5 & 3 & 13,6 & 9 & 40,9 & 5 & 22,7 \\
\hline & & Handball & 1 & 4,5 & 1 & 4,5 & 3 & 13,6 & 12 & 54,5 & 5 & 22,7 \\
\hline & & Basketball & 2 & 9,1 & 0 & 0 & 10 & 45,5 & 10 & 45,5 & 0 & 0 \\
\hline & & Volleyball & 0 & 0 & 0 & 0 & 3 & 13,6 & 8 & 36,4 & 11 & 50,0 \\
\hline & & Individual & 2 & 9,1 & 1 & 4,5 & 4 & 18,2 & 8 & 36,4 & 7 & 31,8 \\
\hline
\end{tabular}

$\mathrm{X} 2=09.078, \mathrm{P}=.133, \mathrm{P}>.05$
No significant difference by branch was found with respect task-oriented leadership behavior. 
www.sstbdergisi.com

International Refereed Academic Journal of Sports, Health and Medical Sciences January / February / March Winter Term Issue: 18 Year: 2016

GEL CODE: M10-M12-M19-M53 ID:268 K:349

ISSN Print: 2146-8508 Online 2147-1711

(ISO 9001-2008 Document No: 12879 \& ISO 14001-2004 Document No: 12880)

(TRADEMARK)

(2015/04315- 2015-GE-18972)

Table 4. \% Distribution of Responses Related to People-Oriented

Leadership Behavior By Branch

\begin{tabular}{|c|c|c|c|c|c|c|c|c|c|c|c|c|}
\hline \multirow{2}{*}{\multicolumn{2}{|c|}{ QUESTION }} & \multirow{2}{*}{ BRANCH } & \multicolumn{2}{|c|}{ Never } & \multicolumn{2}{|c|}{ Seldom } & \multicolumn{2}{|c|}{ Occasionally } & \multicolumn{2}{|c|}{ Frequently } & \multicolumn{2}{|c|}{ Always } \\
\hline & & & $\mathrm{F}$ & $\%$ & $\mathrm{~F}$ & $\%$ & $\mathrm{~F}$ & $\%$ & $\mathrm{~F}$ & $\%$ & $\mathrm{~F}$ & $\%$ \\
\hline \multirow{5}{*}{3} & \multirow{5}{*}{$\begin{array}{l}\text { I would allow members } \\
\text { complete freedom in their } \\
\text { work }\end{array}$} & Football & 5 & 22,7 & 4 & 18,2 & 10 & 45,5 & 3 & 13,6 & 0 & 0 \\
\hline & & Handball & 6 & 27,3 & 7 & 31,8 & 5 & 22,7 & 3 & 13,6 & 1 & 4,5 \\
\hline & & Basketball & 0 & 0 & 11 & 50,0 & 4 & 18,2 & 0 & 0 & 7 & 31,8 \\
\hline & & Volleyball & 3 & 13,6 & 5 & 22,7 & 6 & 27,3 & 8 & 36,4 & 0 & 0 \\
\hline & & Individual & 2 & 9,1 & 3 & 13,6 & 8 & 36,4 & 4 & 18,2 & 5 & 22,7 \\
\hline \multirow{5}{*}{5} & \multirow{5}{*}{$\begin{array}{l}\text { I would permit members to use } \\
\text { their own judgment in solving } \\
\text { problems }\end{array}$} & Football & 3 & 13,6 & 2 & 9,1 & 6 & 27,3 & 6 & 27,3 & 5 & 22,7 \\
\hline & & Handball & 1 & 4,5 & 1 & 4,5 & 9 & 40,9 & 5 & 22,7 & 6 & 27,3 \\
\hline & & Basketball & 0 & 0 & 0 & 0 & 12 & 54,5 & 2 & 9,1 & 8 & 36,4 \\
\hline & & Volleyball & 0 & 0 & 4 & 18,2 & 6 & 27,3 & 12 & 54,5 & 0 & 0 \\
\hline & & Individual & 0 & 0 & 2 & 9,1 & 7 & 31,8 & 4 & 18,2 & 9 & 40,9 \\
\hline \multirow{5}{*}{7} & \multirow{5}{*}{$\begin{array}{l}\text { I would needle members for } \\
\text { greater effort }\end{array}$} & Football & 5 & 22,7 & 3 & 13,6 & 7 & 31,8 & 5 & 22,7 & 2 & 9,1 \\
\hline & & Handball & 2 & 9,1 & 1 & 4,5 & 8 & 36,4 & 7 & 31,8 & 4 & 18,2 \\
\hline & & Basketball & 0 & 0 & 8 & 36,4 & 7 & 31,8 & 5 & 22,7 & 2 & 9,1 \\
\hline & & Volleyball & 0 & 0 & 5 & 22,7 & 0 & 0 & 17 & 77,3 & 0 & 0 \\
\hline & & Individual & 5 & 22,7 & 3 & 13,6 & 7 & 31,8 & 3 & 13,6 & 4 & 18,2 \\
\hline \multirow{5}{*}{10} & \multirow{5}{*}{$\begin{array}{l}\text { I would let members do their } \\
\text { work the way they think best }\end{array}$} & Football & 3 & 13,6 & 0 & 0 & 5 & 22,7 & 11 & 50,0 & 3 & 13,6 \\
\hline & & Handball & 0 & 0 & 2 & 9,1 & 5 & 22,7 & 6 & 27,3 & 9 & 40,9 \\
\hline & & Basketball & 0 & 0 & 0 & 0 & 2 & 9,1 & 12 & 54,5 & 8 & 36,4 \\
\hline & & Volleyball & 0 & 0 & 0 & 0 & 16 & 72,7 & 4 & 18,2 & 2 & 9,1 \\
\hline & & Individual & 0 & 0 & 0 & 0 & 9 & 40,9 & 10 & 45,5 & 3 & 13,6 \\
\hline \multirow{5}{*}{15} & \multirow{5}{*}{$\begin{array}{l}\text { I would turn the members } \\
\text { loose on a job and let them } \\
\text { go to it }\end{array}$} & Football & 1 & 4,5 & 0 & 0 & 0 & 0 & 6 & 27,3 & 15 & 68,2 \\
\hline & & Handball & 0 & 0 & 0 & 0 & 0 & 0 & 13 & 59,1 & 9 & 40,9 \\
\hline & & Basketball & 0 & 0 & 0 & 0 & 0 & 0 & 5 & 22,7 & 17 & 77,3 \\
\hline & & Volleyball & 0 & 0 & 4 & 18,2 & 0 & 0 & 14 & 63,6 & 4 & 18,2 \\
\hline & & Individual & 0 & 0 & 1 & 4,5 & 2 & 9,1 & 8 & 36,4 & 11 & 50,0 \\
\hline \multirow{5}{*}{18} & \multirow{5}{*}{$\begin{array}{l}\text { I would represent the group at } \\
\text { outside meetings }\end{array}$} & Football & 0 & 0 & 1 & 4,5 & 9 & 40,9 & 8 & 36,4 & 4 & 18,2 \\
\hline & & Handball & 0 & 0 & 5 & 22,7 & 9 & 40,9 & 7 & 31,8 & 1 & 4,5 \\
\hline & & Basketball & 2 & 9,1 & 5 & 22,7 & 11 & 50,0 & 4 & 18,2 & 0 & 0 \\
\hline & & Volleyball & 0 & 0 & 0 & 0 & 9 & 40,9 & 7 & 31,8 & 6 & 27,3 \\
\hline & & Individual & 0 & 0 & 5 & 22,7 & 10 & 45,5 & 3 & 13,6 & 4 & 18,2 \\
\hline \multirow{5}{*}{19} & \multirow{5}{*}{$\begin{array}{l}\text { I would be reluctant to allow } \\
\text { the members any freedom of } \\
\text { action }\end{array}$} & Football & 7 & 31,8 & 7 & 31,8 & 5 & 22,7 & 3 & 13,6 & 0 & 0 \\
\hline & & Handball & 4 & 18,2 & 7 & 31,8 & 5 & 22,7 & 6 & 27,3 & 0 & 0 \\
\hline & & Basketball & 7 & 31,8 & 6 & 27,3 & 9 & 40,9 & 0 & 0 & 0 & 0 \\
\hline & & Volleyball & 11 & 50,0 & 5 & 22,7 & 2 & 9,1 & 4 & 18,2 & 0 & 0 \\
\hline & & Individual & 4 & 18,2 & 8 & 36,4 & 6 & 27,3 & 3 & 13,6 & 1 & 4,5 \\
\hline
\end{tabular}


SSTB

www.sstbdergisi.com

International Refereed Academic Journal of Sports, Health and Medical Sciences January / February / March Winter Term Issue: 18 Year: 2016

GEL CODE: M10-M12-M19-M53 ID:268 K:349

ISSN Print: 2146-8508 Online 2147-1711

(ISO 9001-2008 Document No: 12879 \& ISO 14001-2004 Document No: 12880)

(TRADEMARK)

(2015/04315- 2015-GE-18972)

I would let some members have authority that I could keep

24 I would allow the group a high degree of initiative changes

I would trust the group

28 members to exercise good judgment

\begin{tabular}{|c|c|c|c|c|c|c|c|c|c|c|}
\hline Football & 3 & 13,6 & 2 & 9,1 & 8 & 36,4 & 7 & 31,8 & 2 & 9,1 \\
\hline Handball & 0 & 0 & 0 & 0 & 8 & 36,4 & 11 & 50,0 & 3 & 13,6 \\
\hline Basketball & 0 & 0 & 0 & 0 & 11 & 50,0 & 2 & 9,1 & 9 & 40,9 \\
\hline Volleyball & 0 & 0 & 4 & 18,2 & 13 & 59,1 & 2 & 9,1 & 3 & 13,6 \\
\hline Individual & 1 & 4,5 & 1 & 4,5 & 8 & 36,4 & 8 & 36,4 & 4 & 18,2 \\
\hline Football & 1 & 4,5 & 0 & 0 & 6 & 27,3 & 11 & 50,0 & 4 & 18,2 \\
\hline Handball & 1 & 4,5 & 1 & 4,5 & 8 & 36,4 & 8 & 36,4 & 4 & 18,2 \\
\hline Basketball & 2 & 9,1 & 0 & 0 & 10 & 45,5 & 10 & 45,5 & 0 & 0 \\
\hline Volleyball & 0 & 0 & 0 & 0 & 7 & 31,8 & 11 & 50,0 & 4 & 18,2 \\
\hline Individual & 1 & 4,5 & 3 & 13,6 & 10 & 45,5 & 5 & 22,7 & 3 & 13,6 \\
\hline Football & 0 & 0 & 3 & 13,6 & 9 & 40,9 & 3 & 13,6 & 7 & 31,8 \\
\hline Handball & 0 & 0 & 5 & 22,7 & 5 & 22,7 & 9 & 40,9 & 3 & 13,6 \\
\hline Basketball & 0 & 0 & 2 & 9,1 & 0 & 0 & 4 & 18,2 & 16 & 72,7 \\
\hline Volleyball & 0 & 0 & 0 & 0 & 10 & 45,5 & 0 & 0 & 12 & 54,5 \\
\hline Individual & 0 & 0 & 3 & 13,6 & 9 & 40,9 & 5 & 22,7 & 5 & 22,7 \\
\hline Football & 0 & 0 & 3 & 13,6 & 7 & 31,8 & 6 & 27,3 & 6 & 27,3 \\
\hline Handball & 0 & 0 & 0 & 0 & 12 & 54,5 & 4 & 18,2 & 6 & 27,3 \\
\hline Basketball & 0 & 0 & 0 & 0 & 9 & 40,9 & 7 & 31,8 & 6 & 27,3 \\
\hline Volleyball & 2 & 9,1 & 0 & 0 & 9 & 40,9 & 11 & 50,0 & 0 & 0 \\
\hline Individual & 0 & 0 & 0 & 0 & 9 & 40,9 & 6 & 27,3 & 7 & 31,8 \\
\hline Football & 0 & 0 & 0 & 0 & 4 & 18,2 & 14 & 63,6 & 4 & 18,2 \\
\hline Handball & 3 & 13,6 & 1 & 4,5 & 9 & 40,9 & 6 & 27,3 & 3 & 13,6 \\
\hline Basketball & 2 & 9,1 & 0 & 0 & 2 & 9,1 & 8 & 36,4 & 10 & 45,5 \\
\hline Volleyball & 0 & 0 & 2 & 9,1 & 3 & 13,6 & 13 & 59,1 & 4 & 18,2 \\
\hline Individual & 0 & 0 & 1 & 4,5 & 5 & 22,7 & 6 & 27,3 & 10 & 45,5 \\
\hline Football & 7 & 31,8 & 6 & 27,3 & 6 & 27,3 & 1 & 4,5 & 2 & 9,1 \\
\hline Handball & 10 & 45,5 & 0 & 0 & 5 & 22,7 & 3 & 13,6 & 4 & 18,2 \\
\hline Basketball & 13 & 59,1 & 2 & 9,1 & 4 & 18,2 & 0 & 0 & 3 & 13,6 \\
\hline Volleyball & 5 & 22,7 & 8 & 36,4 & 3 & 13,6 & 4 & 18,2 & 2 & 9,1 \\
\hline Individual & 9 & 40,9 & 6 & 27,3 & 5 & 22,7 & 2 & 9,1 & 0 & 0 \\
\hline Football & 1 & 4,5 & 2 & 9,1 & 3 & 13,6 & 6 & 27,3 & 10 & 45,5 \\
\hline Handball & 3 & 13,6 & 0 & 0 & 1 & 4,5 & 11 & 50,0 & 7 & 31,8 \\
\hline Basketball & 0 & 0 & 0 & 0 & 4 & 18,2 & 3 & 13,6 & 15 & 68,2 \\
\hline Volleyball & 0 & 0 & 2 & 9,1 & 2 & 9,1 & 14 & 63,6 & 4 & 18,2 \\
\hline Individual & 0 & 0 & 1 & 4,5 & 1 & 4,5 & 8 & 36,4 & 12 & 54,5 \\
\hline
\end{tabular}

$\mathrm{X} 2=12.110, \mathrm{P}=.088, \mathrm{P}>.05$

No significant difference by branch was found with respect task-oriented leadership behavior.

\section{DISCUSSION}

An examination of the leadership behavior of the students from the department of coaching education demonstrates that they generally fit both task-oriented and people-oriented types of leadership, and that there is no significant difference between the genders. 


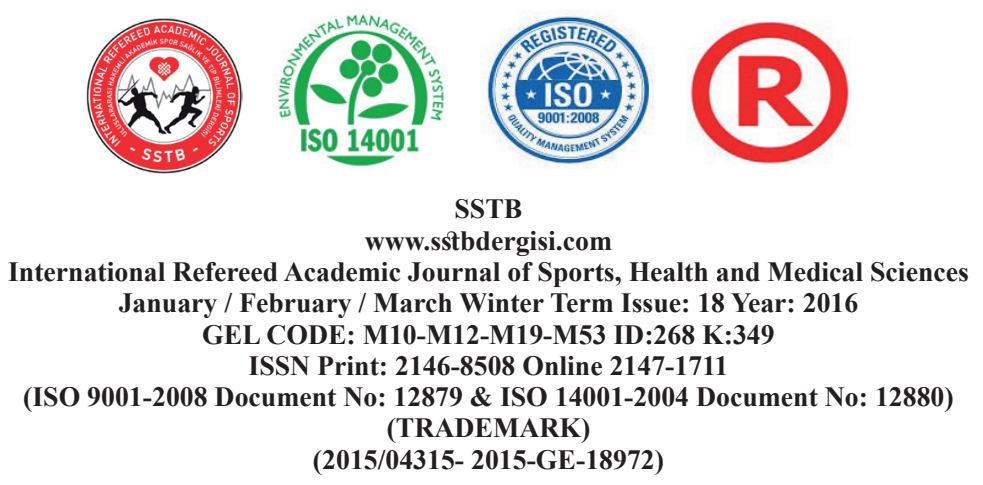

In the leadership scale developed by Richard Daft (2008), the questions 1, 2, 4, 6, 8, 9, 11, $12,13,14,16,17,20,21,23,25,27,29,30$, 31 and 33 support task-oriented leadership. An examination of the students' leadership behavior by gender shows that they generally fit task-oriented type of leadership, and that there is no difference between the genders.

Kattat et al. (2004) determined that there was no difference by gender in the work-related leadership behavior of the general secretaries working for sports federations. Durukan et al. (2006) found no significant difference in their study comparing the leadership behaviors (establishing the structure) of female and male students of the schools of physical education and sports. In his study related to the leadership types of sports managers that are active at different levels of sports management, Gökçe (2005) concluded that there was no difference between genders. These studies are supportive of our study.

An examination of the leadership behavior of the coaching education students by branch indicates that they generally fit both taskoriented and people-oriented types of leadership, but there is difference between some branches in certain cases. This difference may be resulting from the characteristic features of the branches.

In our study, the volleyball branch responded "always" to the question "I would encour- age overtime work"; handball and basketball branches responded "always" to the question "I would encourage the use of uniform procedures"; the handball branch responded "always" to the question "I would stress being ahead of competing groups", and these branches thus differed from other branches in these respects.

The resources we have reached through a review of the literature are supportive of our results, emphasizing that coaches exhibit both task-oriented and people-oriented types of leadership and stating that such leadership style facilitates success in sports (Davis 97, Temel 2010, Didari et al. 2008).

The basketball branch responded "seldom" to the question "I would try out my ideas in the group"; the volleyball branch responded "frequently" to the question "I would tolerate postponement and uncertainty"; the basketball branch responded "seldom" to the question "I would speak for the group if visitors were present" and these branches thus differed from other branches in these respects.

According to the theory of behavior, any person can be a leader by learning the behavior of an efficient leader. Therefore, leadership is not innate (Tiryaki, 2000). The main idea of the theory attempting to explain the process of leadership is that what makes leaders successful and efficient is not their characteristics but the behavior they exhibit in 


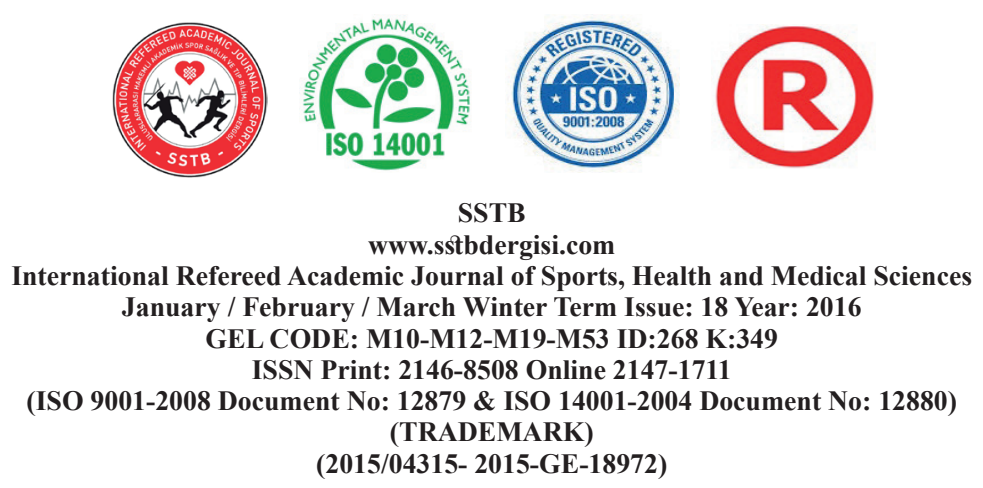

performing leadership. Leaders' way of communication with their subordinates, whether they give some powers to them or not, their way of planning and controlling, their way of determining the goals, and such other behavior have been considered as important factors determining the efficiency of leaders. Therefore, this theory has laid as much emphasis on the group members, who are the secondary variable, as it has laid on the leaders themselves (Koçel 1999).

According to Tamer and Pulur (2001), a recently-graduated physical education teacher confront such problems as overcrowded classrooms, poor environment, indifferent families, poor economic conditions, spoiled children, indifference of some students, students' unpreparedness for the classes, and absence as well as a great problem of discipline arising from the young appearance of the teacher and some students' desire to test him/ her. Therefore, this situation can be expected to cause the young teachers to exhibit a more autocratic/oppressive behavior towards students in order to overcome this problem of discipline. However, young teachers' not exhibiting autocratic/oppressive behavior indicates that they have received appropriate education during their undergraduate education.

In their study examining the relation between the productivity and leadership styles of managers in public sports organizations, Didari et al. (2008) determined that there was no relation between productivity and leadership styles in people-centered and task-centered dimensions. It was determined, however, that leaders in public sports organizations should exhibit leadership behavior in both peoplecentered and task-centered dimensions in order to be effective.

A study on leadership behavior carried out in Ohio State University has shown that the pace of labor turnover and the level of absence (lack of attendance) decrease as the leaders' behavior of taking people into consideration increases. It has been also determined that an increase in structure-initiating behavior increases the success and performance of group members (Ataman, 2001). The two important universities with respect to behavioral approaches, Ohio and Michagen Universities, have been criticized in some aspects. Among the main aspects that are criticized are the limitation of leadership behavior to two dimensions, proposition of a leadership style presumed to be accepted as universally effective, and the fact that situational variables are not taken into consideration (Erçetin, 2000).

\section{CONCLUSION and RECOMMENDA- TIONS}

Since the concept of leadership is a personality trait, the students studying in the departments of coaching education and other departments of the Schools of Physical Edu- 


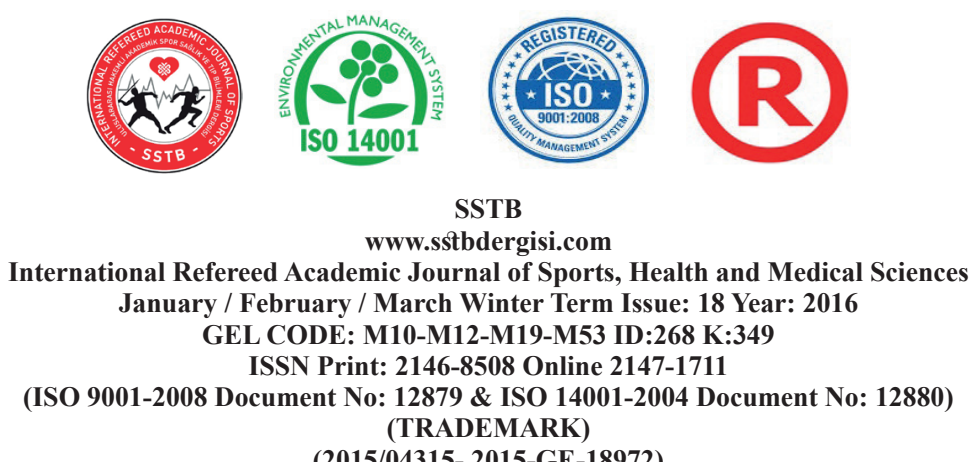

(2015/04315- 2015-GE-18972)

cation and Sports should be assigned responsibilities aimed at improving their leadership skills starting from their first years in these schools. It should be noted that the sports activities are one of the most important environments in which leadership characteristics develop. The results of this study confirm the idea that the students of the Department of Coaching Education have leadership orientations. It is thought that inclusion of practical activities in addition to theoretical courses in the curricula will contribute to the improvement of leadership characteristics.

\section{REFERENCES}

ATAMAN, G., (2002). İşletme Yönetimi Temel Kavramlar \& Yeni Yaklaşımlar, 2. Bask1, Türkmen Kitabevi, İstanbul

AYDIN, M., (1994). Eğitim Yönetimi, Hatipoğlu Yayınevi. Ankara, p. 233

DAFT, R.L., (2008). Management, Eighth Edition, Thomson Higher Education, USA

DAVIS, K.A., (1997). Sport management. Iowa: Brown Benchmark Publishers

DIDARI, J., et all., (2008). The Relationship Between Leadership Style And Productivity in Public Sport, Book Of Abstracts, 16th EASM European Sport Management Conference, 10-13 September, Heidelberg / Bayreuth, 429-431
DONUK, B., (2007). Liderlik ve Spor, p.19, Ötüken Yayınevi, İstanbul

DURUKAN, E., et all., (2006). Selçuk Üniversitesi Beden Eğitimi ve Spor Yüksekokulu Öğrencilerinin Cinsiyete Bağlı Olarak Liderlik Davranışı (Yapıyı Kurma Boyutu) Yönünden Karşılaştırılması, Gazi University Kırşehir Faculty of Education, Volume 6, No 1, 25-32

ELMUTI, D., et all., (2005). Does Education Have a Role in Developing Leadership Skills? Management Decision, Vol. 43, No. $7 / 8$, p. $43-56$

ERÇETIN , Ş., (2000). "Lider Sarmalında Vizyon". Nobel Yayınları, 2nd Edition, Ankara

GELATT, J.P., (2002). “Leadership". Mann, C.J. and Götz, K. (Ed.). The Development of Management Theory and Practice in the United States (65-86). USA: Pearson Custom Publishing

GÖKÇE, Z., (2005). Spor Yönetiminin Farkl1 Boyutlarında Yer Alan Spor Yöneticilerinin Liderlik Tiplerinin Araştırılması (Ege Bölgesi Örneği), Celal Bayar University Institute of Health Sciences Master's Thesis

JAMES, J., et all., (2001). Uzun Sapl1 Gelincik, BZD Yayınları, İstanbul 


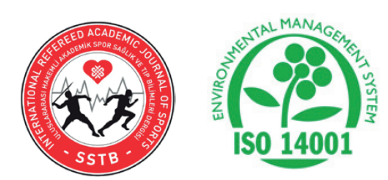

SSTB

www.sstbdergisi.com

International Refereed Academic Journal of Sports, Health and Medical Sciences

January / February / March Winter Term Issue: 18 Year: 2016

GEL CODE: M10-M12-M19-M53 ID:268 K:349

ISSN Print: 2146-8508 Online 2147-1711

(ISO 9001-2008 Document No: 12879 \& ISO 14001-2004 Document No: 12880)

(TRADEMARK)

(2015/04315- 2015-GE-18972)

KATTAT, D., et all., (2004). Spor Federasyonlarında Görev Yapan Genel Sekreterlerin Liderlik Analizi, 10. Uluslararas1 Spor Bilimleri Kongresi Özet Kitabı, Antalya, p. 96

KOÇEL, T., (1999). İşletme Yöneticiliği, Beta Basın Yayın Dağıtım, Istanbul

Konter, E., (1996). Bir Lider Olarak Antrenör, Alfa Basım Yayım Dağıtım, İstanbul

LAURENT, et all., (2007). Leadership Behaviors of Athletic Training Leaders Compared With Leaders in Other Fields, Journal of Athletic Training, 42(1):120-125

MOLE, G., (2004). Can Leadership Be Taught?, Leadership in Organizations, Editor; John Storey, Routledge, p. 127, London
NORTHOUSE, G., P., (2004). Leadership: Theory and Practice, 3rd Ed., Sage Publications, London

SABUNCUOĞLU, Z., TÜZ, M., (2001). Örgütsel Psikoloji, Ezgi Kitabevi, Bursa, p. 217

TEMEL, V., (2010). "Konya İline Ait Individual ve Takım Sporu Antrenörlüğü Yapan Bireylerin Liderlik Tarzlarının Karşılaştırılması" Master's Thesis, Karamanoğlu Mehmetbey University Institute of Social Sciences, Karaman

TAMER, K., et all., (2001). Beden Eğitimi ve Sporda Öğretim Yöntemleri. Ada Matbaacilık, Ankara

TIRYAKI, Ş., (2000). Spor Psikolojisi-Kavramlar, Kuramlar ve Uygulama, Eylül Yayınları, Ankara 